\title{
Polychondritis: Eine neue Erkrankung beim Braunvieh?
}

\author{
Bleul, U ; Stranzinger, G ; Sydler, T
}

\begin{abstract}
In den letzten 10 Jahren sind immer wieder Tiere der Rasse Braunvieh mit Veränderungen der Ohrmuscheln aufgetreten. Bei einigen dieser Tiere waren auch eine verkürzte und verdickte Epiglottis und Veränderungen der Aryknorpel endoskopisch feststellbar. Histopathologisch war in den meisten Fällen der elastische Knorpel durch ein Knorpelregenerat aus Faserknorpel und hyalinem Knorpel ersetzt. Ursache und genaue Pathogenese dieser Veränderungen konnten nicht eruiert werden.
\end{abstract}

DOI: https://doi.org/10.1024/0036-7281/a000265

Other titles: Polychondritis: A new disease in Swiss Braunvieh?

Posted at the Zurich Open Repository and Archive, University of Zurich

ZORA URL: https://doi.org/10.5167/uzh-52862

Journal Article

Accepted Version

Originally published at:

Bleul, U; Stranzinger, G; Sydler, T (2011). Polychondritis: Eine neue Erkrankung beim Braunvieh? Schweizer Archiv für Tierheilkunde, 153(11):526-529.

DOI: https://doi.org/10.1024/0036-7281/a000265 


\title{
1 Polychondritis: eine neue Erkrankung beim Braunvieh?*
}

2

3

5

6

7

8

\author{
U. Bleul ${ }^{1}$, G. Stranzinger ${ }^{2}$, T. Sydler ${ }^{3}$
}

${ }^{1}$ Klinik für Fortpflanzungsmedizin, ${ }^{2}$ Institut für Nutztierwissenschaften und ${ }^{3}$ Institut für Veterinärpathologie der Universität Zürich

\section{Zusammenfassung}

In den letzten 10 Jahren sind immer wieder Tiere der Rasse Braunvieh mit Veränderungen der Ohrmuscheln aufgetreten. Bei einigen dieser Tiere waren auch eine verkürzte und verdickte Epiglottis und Veränderungen der Aryknorpel endoskopisch feststellbar. Histopathologisch war in den meisten Fällen der elastische Knorpel durch ein Knorpelregenerat aus Faserknorpel und hyalinem Knorpel ersetzt. Ursache und genaue Pathogenese dieser Veränderungen konnten nicht eruiert werden.

Schlüsselwörter: Rind, Elastischer Knorpel, Ohrmuschel, Larynx, Chondritis

\section{Polychondritis: a new disease in Swiss Braunvieh?}

\section{Summary}

Over the last 10 years Swiss Braunvieh cattle with malformations of the pinnae have repeatedly been reported. Endoscopy revealed a shortened and thickened epiglottis and malformations of the arytenoid cartilage in some of these animals. In most cases the elastic cartilage was replaced by fibrocartilage and hyaline cartilage. The direct cause and pathogenesis of the malformations could not be determined.

Keywords: Bovine, elastic cartilage, pinna, larynx, chondritis

\section{Einleitung}

In den Jahren 2000 und 2001 wurden im Departement für Nutztiere der Universität Zürich zwei Braunviehkühe mit Veränderungen der Ohren in Form von herabhängenden Ohrenmuscheln und einer Verdickung der Ohrbasis mit mehr oder weniger vollständigem Verschluss des äusseren Gehörgangs vorgestellt und untersucht (Bleul et al., 2006). Diese Veränderungen waren bisher unbekannt und konnten weder klinisch noch pathologisch 
1 einem Erkrankungskomplex zugeordnet werden. Histologisch zeigte der Knorpel der

Ohrbasis eine Verdickung desselben als Folge von proliferiertem Faserknorpel und hyalinem Knorpel. Elastischer Knorpel war nicht mehr vorhanden. Die Knorpelproliferation war von einer Proliferation eines reifen, Gefässe enthaltenden Bindegewebes begleitet, welche sowohl vom Perichondrium her als auch zentral im proliferierten Knorpel stattfand. Eine Entzündung war nur minimal in Form weniger perivaskulär gelegener Lymphozyten vorhanden. Das Karyogramm der beiden Tiere zeigte bei normaler Chromosomenzahl eine Differenz in der Länge der langen Arme der X-Chromosome, weshalb ein Zusammenhang mit den Ohranomalien vermutet wurde.

In den folgenden Jahren wurden aus allen Teilen der Schweiz weitere Tiere mit den gleichen phänotypischen Anzeichen gemeldet (Abb. 1). Bei 10 von diesen wurden weitergehende klinische, zytogenetische, endoskopische und pathologische Abklärungen durchgeführt.

\section{Material und Methoden}

\section{Tiere}

Es wurden 7 weibliche und 3 männliche Rinder der Rasse Braunvieh im Alter zwischen 6 Wochen und 5 Jahren untersucht. Alle Tiere zeigten die typischen hängenden Ohrmuscheln und eine Verdickung der Ohrbasis. Vier Tiere gingen anschliessend an die Untersuchungen wieder zurück in den Ursprungsbetrieb, 2 wurden nach den Abklärungen geschlachtet und seziert und ein männliches und 3 weibliche Tiere wurden zur Beobachtung eingestallt.

\section{Zytogenetische Analysen}

Alle Tiere wurden einer eingehenden klinischen Untersuchung unterzogen. Anschliessend wurde allen Tieren eine Muskelbiopsie aus dem Triceps brachii entnommen, daraus Fibroblasten kultiviert und an diesen zytogenetische Untersuchungen durchgeführt (Ahrens, 2004; Stranzinger, 1991). Die Metaphasen aller Tiere wurden mit Giemsa gefärbt, bei 4 Tieren erfolgte zusätzlich eine G-Bänderung und bei 2 weiteren eine Q-Bänderung (Ahrens, 2004). Anhand der Giemsa gefärbten Metaphasen erfolgte die Karyotypisierung und bei den weiblichen Tieren die Identifikation der X-Chromosomen zum Längenvergleich deren langen Arme (Abb. 2). Um präparations- und vergrösserungsbedingte Längenunterschiede auszugleichen, wurden in 55 Metaphasen jeweils die langen und kurzen Arme am Computer mit einem Messprogramm (NIH Image, http://rsb.info.nih.gov/nih-image/) ausgemessen und

*Herrn Prof. Dr. Dr. h. c. Ueli Braun zum 60. Geburtstag gewidmet 
1 das Verhältnis der beiden berechnet. Zum Vergleich mit Karyogrammen von gesunden 2 Kühen wurden 35 Giemsa-gefärbte Metaphasen von Schlachtkühen hergestellt und 3 ausgemessen. Die Vergleiche der Längenverhältnisse der beiden X-Chromosomen einer 4 jeweiligen Metaphase und die zwischen den kleineren bzw. grösseren X-Chromosomen der gesunden und erkrankten Tiere wurde mittels gepaartem t-Test oder t-Test für 2 Stichproben berechnet. Ein $\mathrm{P}<0.05$ wurde als signifikant festgelegt.

\section{Endoskopie}

Bei 4 Tieren wurde aufgrund des bei der klinischen Untersuchung festgestellten Hustens eine Endoskopie der Atemwege mit einem flexiblen Videoendoskop (Storz, Tuttlingen, Deutschland) durchgeführt. Hierzu wurde nach einer Sedation $(0.02 \mathrm{mg} / \mathrm{kg}$ Körpergewicht Xylazin i. v., Xylazin, Streuli Pharma) das Endoskop transnasal in den Larynx und die Trachea vorgeschoben. In einem Zeitraum von einer Woche bis zu 2 Jahren nach der Untersuchung wurden diese 4 Tiere geschlachtet und die inneren Organe wurden makro- und histopathologisch untersucht. Gewebeproben wurden nach Standardmethoden fixiert, in Paraffin eingebettet und die Beurteilung erfolgte am Hämatoxilin-Eosin gefärbten Schnitt.

\section{Ergebnisse}

Anamnestisch wiesen alle Tiere ein plötzlich auftretendes Herunterhängen der Ohrmuscheln auf, wobei meist erst eine und 1 bis 2 Wochen später die andere Ohrmuschel herunterhing. Das jüngste Tier war zu diesem Zeitpunkt 6 Wochen alt, das älteste 3 Jahre. Die Besitzer berichteten von keiner erkennbaren Beeinträchtigung der Tiere. Auch in der klinischen Untersuchung wurden neben den Veränderungen der Ohrmuscheln keine Auffälligkeiten festgestellt. Die Ohrbasis beider Ohren war bei allen untersuchten Tieren mit 2.5 bis $4 \mathrm{~cm}$ so verdickt, dass der äussere Gehörgang teilweise oder vollständig verlegt wurde (Abb. 1b). Während die Umfangsvermehrung bei den älteren Tieren von hart-elastischer Konsistenz war, konnte bei 2 Kälbern im Alter von 6 und 8 Wochen flüssiger Inhalt in der Zubildung palpiert werden. Keines der Tiere zeigte bei der Palpation eine Schmerzreaktion. Ebenso waren die lateralen Anteile der Ohrmuschel, auch im Bereich der Ohrmarken, ohne Auffälligkeiten.

Alle untersuchten Tiere wiesen ein normales bovines Karyogramm $(2 n=60)$ auf. In den mit Giemsa gefärbten Metaphasen konnte ein signifikanter Grössenunterschied der beiden X-

*Herrn Prof. Dr. Dr. h. c. Ueli Braun zum 60. Geburtstag gewidmet 
1 Chromosomen festgestellt werden, der aber auch in den Metaphasen der Kontrolltiere 2 vorhanden werden. Die angewandten Bänderungsfärbungen zeigten keinen Unterschied 3 zwischen den X-Chromosomen.

4 Vier Tiere wurden untereinander und mit anderen Tieren angepaart. Keines der 23 Nachkommen zeigte die entsprechenden Veränderungen der Ohrmuscheln. Eine Kuh und ein Stier, die zur Zucht genutzt wurden, zeigten 1 bzw. 2 Jahre nach der Erstuntersuchung vermehrten Husten, insbesondere während und nach der Futteraufnahme. Daraufhin wurde bei diesen beiden und 2 weiteren Tieren eine Endoskopie der oberen Luftwege durchgeführt. Bei 3 der 4 Tiere konnte während der Untersuchung eine temporäre oder dauerhafte Dorsalverlagerung des weichen Gaumens aufgrund einer verkürzten und verdickten Epiglottis festgestellt werden. Daneben war beim Stier und der Kuh sowie bei einem 2 Monate alten Kalb eine deutliche Verkleinerung und Asymmetrie der Processi corniculati der Aryknorpel erkennbar (Abb. 3a).

Bei den adulten Tieren war histopathologisch an der Ohrmuschelbasis an Stelle eines kompakten elastischen Knorpels ein Knorpelgebilde aus multifokal-konfluierendem Faserknorpel und hyalinem Knorpel mit unregelmässiger Grenze zum Perichondrium und zentraler Bindegewebsproliferation getreten. Die zwei Kälber mit palpierbarer Fluktuation an der verdickten Ohrbasis zeigten histologisch eine Koagulationsnekrose von elastischem Knorpel mit vorwiegend lympho-histiozytärer Entzündung mit mehrkernigen Riesenzellen vom Fremdkörpertyp. Bei den verdickten und verkürzten Kehldeckeln und den nur noch rudimentär vorhanden Processi corniculati der Aryknorpel (Abb. 3b) war der elastische Knorpel ebenfalls durch Proliferation von hyalinem Knorpel und Faserknorpel ersetzt. Bei zwei Tieren waren noch Gebiete mit degeneriertem elastischen Knorpel und bei einem Tier eine herdförmige granulomatöse Entzündung als Reaktion auf eine Nekrose von elastischem Knorpel vorhanden.

\section{Diskussion}

Formanomalien der Ohrmuscheln sind bei einer Vielzahl von Tierarten und beim Menschen beschrieben. Dabei kann es sich um angeborene Missbildungen oder die Folge eines Traumas oder einer Entzündung handeln. Während beim Rind hauptsächlich angeborene

*Herrn Prof. Dr. Dr. h. c. Ueli Braun zum 60. Geburtstag gewidmet 
1 Kerben in der Ohrmuschel beschrieben wurden (Lush, 1922; MacDonald, 1957; Wriedt, 1925), sind beim Menschen zahlreiche angeborene Veränderungen des Aussenohrs bekannt. worden (Melnick et al., 1979). Sie beruhen in vielen Fällen auf einer chromosomalen Störung, wie zum Beispiel dem Turner-Syndrom, bei dem entweder der Verlust eines Teils oder das völligen Fehlen des X-Chromosoms vorliegt (Barrenas et al., 2000). Bei den ersten untersuchten Rindern mit verdickten Ohrbasen und hängenden Ohren wurde ebenfalls eine chromosomale Ursache in Form einer terminalen Erosion oder einer minimen Translokation der langen Arme bei einem der beiden X-Chromosomen mit einem Autosom vermutet (Bleul et al., 2006). Durch verschiedene Bänderungsfärbungen und dem Vergleich der Längenunterschiede der langen Arme der X-Chromosomen mit gesunden Kontrolltieren konnte dieser Verdacht aber nicht bestätigt werden. Die Längenunterschiede könnten auch die Folge der Inaktivierung eines der beiden X-Chromosome bei weiblichen Rindern sein (Knogl, 1975). Jedoch ist nicht auszuschliessen, dass die beschriebenen Veränderungen auch eine andere genetische Grundlage haben können.

Bei 3 Tieren konnte die entzündliche Zerstörung des elastischen Knorpels nicht nur an der Ohrmuschel, sondern auch am Larynx festgestellt werden. Es liegt somit eine multizentrische Pathogenese mit Polychondritis von elastischem Knorpel vor. Als multizentrisches Geschehen tritt beim Menschen die „relapsing polychondritis" auf, eine seltene Autoimmunerkrankung, bei der häufig der elastische Knorpel jedoch auch der hyaline Knorpel betroffen sind (Belot et al., 2010; Lahmer et al., 2010). Auch bei der Katze ist eine ähnliche Erkrankung beschrieben (Gerber et al., 2002). Eine Autoimmunerkrankung kann bei den hier untersuchten Tieren ebenfalls nicht ausgeschlossen werden. Auslöser dafür könnte ein toxisches, chemisches oder infektiöses Agens sowie ein Trauma sein (Lahmer et al., 2010), durch den eine autoimmunbedingte Entzündung der Knorpel an unterschiedlichen Lokalisationen hervorgerufen wird. So zeigten Ratten, denen Ohrmarken in ein Ohr eingesetzt wurden, an beiden Ohren Knorpelveränderungen, die denen der hier untersuchten Tiere sehr ähnlich sind (Kitagaki et al., 2003). In wieweit das Krankheitsbild beim Rind dem der "relapsing polychondritis" beim Menschen entspricht, bedarf weiterer Abklärungen. Insbesondere der rezidivierende und autoimmune Charakter sind beim Rind bisher nicht nachgewiesen, ebenso konnte eine Beteiligung von hyalinem Knorpel nicht beobachtet werden.

*Herrn Prof. Dr. Dr. h. c. Ueli Braun zum 60. Geburtstag gewidmet 


\section{Literatur}

Ahrens E., Comparative chromosomal studies of E. caballus and E. przewalskii in a F1 hybrid. Dissertation Universität Zürich, 2004.

Barrenas M.-L., Landin-Wilhelmsen K., Hanson C.: Ear and hearing in relation to genotype and growth in Turner syndrome. Hearing Res. 2000, 144: 21-28.

Belot A., Duquesne A., Job-Deslandre C., Costedoat-Chalumeau N., Boudjemaa S., Wechsler B., Cochat P., Piette J.-C., Cimaz R.: Pediatric-Onset Relapsing Polychondritis: Case Series and Systematic Review. J. Pediatr. 2010, 156: 484-489.

Bleul U., Ahrens E., Stranzinger G., Sydler T., Ohlerth S., Braun U.:Auricular chondropathy in two Swiss Braunvieh heifers. Vet. Rec. 2006, 159: 890-892.

Gerber B., Crottaz M., von Tscharner C., Scharer V.: Feline relapsing polychondritis: two cases and a review of the literature. J. Feline Med. Surg. 2002, 4: 189-194.

Kitagaki M., Suwa T., Yanagi M., Shiratori K.: Auricular chondritis in young ear-tagged Crj:CD(SD)IGS rats. Lab. Anim. 2003, 37: 249-253.

Knogl R.: X - Chromosomenpolymorphismus beim weiblichen Rind. Diplomhausarbeit, TUM München, 1975.

Lahmer T., Treiber M., von Werder A., Foerger F., Knopf A., Heemann U., Thuermel K.: Relapsing polychondritis: An autoimmune disease with many faces. Autoimmun. Rev. 2010, 9: 540-546.

Lush L.J.: An hereditary notch in the ears of Jersey cattle. J. Hered. 1922, 13: 8-13.

MacDonald M.A.: Notched ears in New Zealand dairy cattle. J. Hered. 1957, 48: 244-247.

Melnick M., Myrianthopoulos N.C., Paul N.W.: External ear malformations: epidemiology, genetics, and natural history. Birth Defects: Original Article Series 1979, 15: i-ix.

Stranzinger G.: Genetische Grundlagen, Zellgenetik, In: Brem, G., Kräusslich, H., Stranzinger, G. (Eds.) Experimentelle Genetik in der Tierzucht. Eugen Ulmer Verlag, Stuttgart 1991: 78-106.

Wriedt C.: Vererbliche Scharten an den Ohren des Rindes. Z. Tierz. Züchtungsbio. 1925, 3: 235-238.

\section{Korrespondenz}

Ulrich Bleul

Klinik für Fortpflanzungsmedizin

Departement für Nutztiere

Vetsuisse-Fakultät Universität Zürich

Winterthurerstrasse 260

$\mathrm{CH}-8057$ Zürich

E-Mail: ubleul@vetclinics.uzh.ch

Tel.: + 41 (0)44 6358651

Fax: + 41 (0)446358908

*Herrn Prof. Dr. Dr. h. c. Ueli Braun zum 60. Geburtstag gewidmet 
2 Abbildung 1: (a) Drei Monate altes Braunviehkalb mit beidseitiger Verformung der

3 Ohrmuschelbasis. (b) Ohrmuschel des gleichen Kalbes mit vollständigem Verschluss des

4 äusseren Gehörgangs.

5

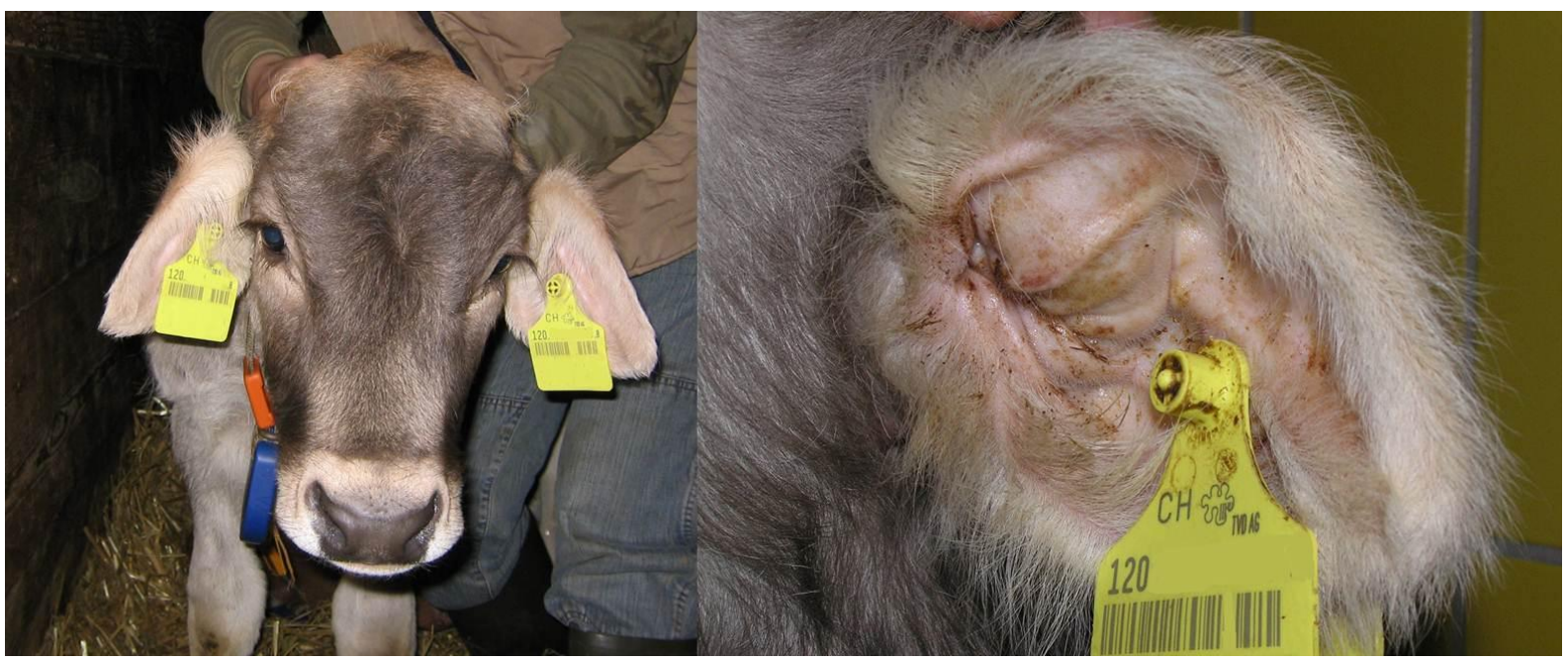

7

8

9 Abbildung 2: Ausschnitt aus einem Karyogramm einer Kuh mit Polychondritis. Zur

10 Berechnung der Längenunterschiede der langen Arme der X-Chromosomen (Pfeil) wurde das

11 Verhältnis der langen zu den kurzen Armen gebildet ( $\$$ ).

*Herrn Prof. Dr. Dr. h. c. Ueli Braun zum 60. Geburtstag gewidmet 
1

2

3

4

5

6

7

8

9

10

11

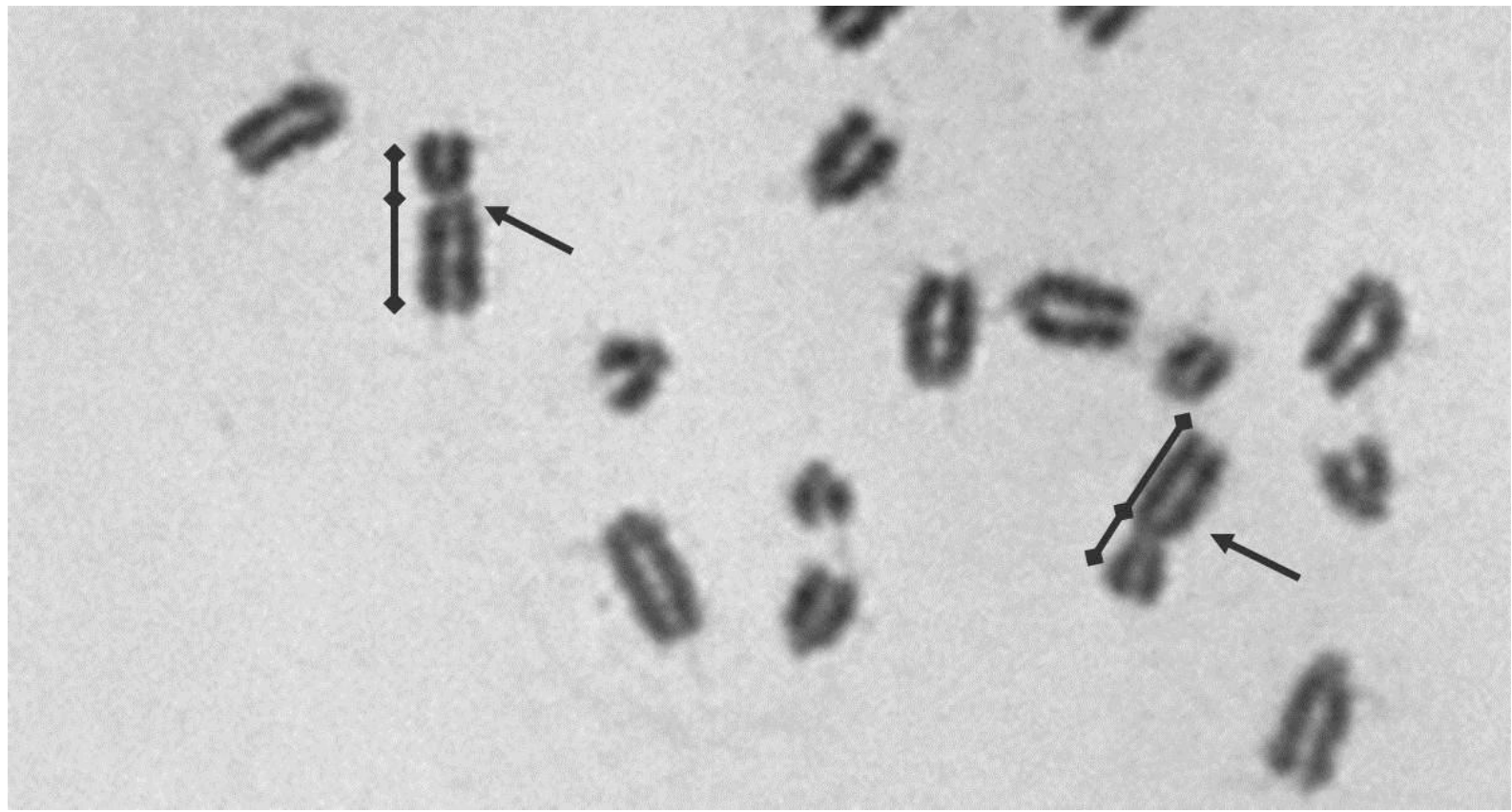

4 Abbildung 3: (a) Endoskopisches Bild des Larynx von rostal einer fünfjährigen Braunviehkuh .

5 Die Epiglottis ist durch den weichen Gaumen $(X)$ verdeckt und die Stimmritze ist sichtbar

(Pfeil). Der rechte Processus corniculatus (RPC) ist deutlich kleiner als der linke (LPC). (b)

7 Sektionspräparate eines Larynx eines 2 Monate alten Braunviehkalbes mit Polychondritis

8 (oben) und eines Vergleichskalbes im selben Alter (unten). Beim erkrankten Larynx oben

fehlt der Processus corniculatus (PC) des Aryknorpels (A) vollständig und die Epiglottis (E) ist 10 verdickt. 

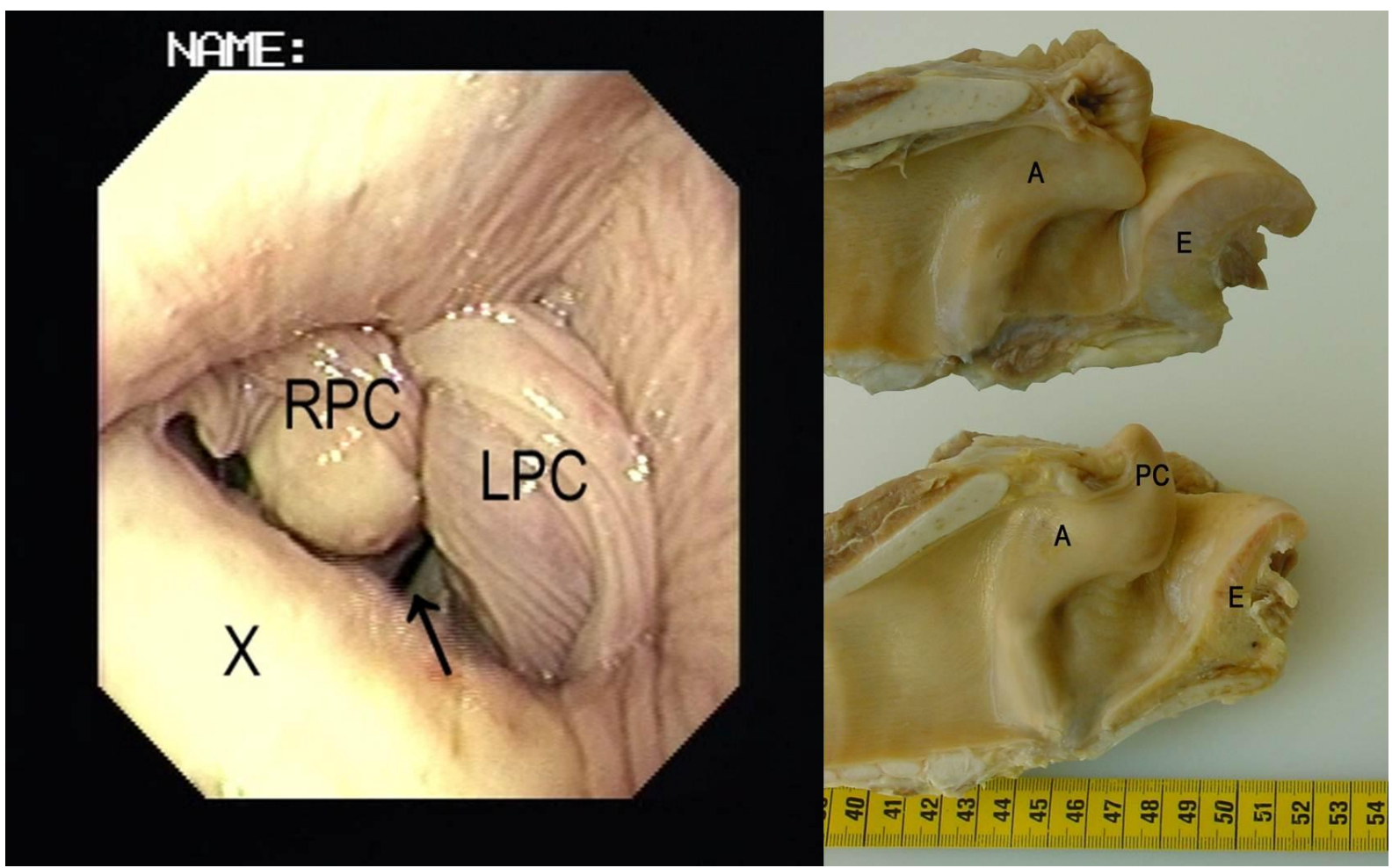

*Herrn Prof. Dr. Dr. h. c. Ueli Braun zum 60. Geburtstag gewidmet 